\title{
Características socioeconómicas y sociodemográficas de las familias de pacientes hospitalizados en el Instituto Nacional de Salud San Borja, 2018
}

Isabel Andrea Gantu Palacios*1,a; Giovana Genoveva Ccasani Huamán 2,b; Ana Francisca Pérez Solis 2,b

\section{RESUMEN}

Objetivo: Describir las características familiares y sociales de los pacientes hospitalizados en el Instituto Nacional de Salud del Niño San Borja durante los meses de abril a diciembre de 2018.

Materiales y métodos: Investigación descriptiva, observacional y analítica. La selección de la muestra fue probabilística de un universo de 4383 pacientes. La muestra fue de 573 pacientes, con un nivel de confianza de $96 \%$, y un margen de error de 4,00 \%. El instrumento empleado fue la ficha de recolección de datos de la historia clínica. La información se procesó con el paquete estadístico SPSS.

Resultados: El 47,12 \% de los pacientes proceden de Lima Metropolitana; el 2,97 \%, de la Provincia Constitucional del Callao, y el 49,91\% del interior del país, lo que incluye Lima Provincia.

El 73,65 \% de pacientes vive en condición de pobreza; el 24,26 \% tiene condición de pobreza extrema; y solo el 2,09 \% son considerados no pobres. El 40,49\% de pacientes habita en viviendas en condiciones inadecuadas. La mayoría de los participantes son beneficiarios del Seguro Integral de Salud (SIS) (94,24\%). Respecto al nivel educativo de las madres, el 1,92 \% no tiene instrucción y el 7,68 \% no concluyeron los estudios primarios. El 0,52 \% de los padres no tienen instrucción, y el 4,19\% tienen estudios primarios incompletos. En cuanto a la educación básica regular (EBR), 43,11\% de las madres y $39,3 \%$ de los padres la concluyeron; mientras que solo $9,77 \%$ del grupo masculino y $6,63 \%$ del femenino cuentan con educación superior técnica.

Conclusiones: El estudio permitió un acercamiento a las condiciones socioeconómicas de los pacientes, en tanto determinantes de la salud. Se evidencia que la mayoría de pacientes viven en condición de pobreza y pobreza extrema. Un porcentaje significativo procede de los diferentes departamentos del país, lo que refleja el centralismo nacional en cuanto a los centros pediátricos especializados y, a la vez, evidencia la importancia del enfoque de interculturalidad en salud. El tipo de seguro de salud está relacionado con la condición económica: se corrobora que la mayoría es beneficiaria del Seguro Integral de Salud (SIS) y vive en condición de pobreza y pobreza extrema. La condición económica del paciente guarda relación con la proporción de viviendas que no cumplen con los requerimientos mínimos de habitabilidad, lo cual es un factor de riesgo para pacientes con enfermedades crónicas o postrasplantados (médula ósea y órganos sólidos). Finalmente, los padres de familia muestran un mayor nivel de instrucción que las madres, lo cual podría sugerir una brecha de género.

Palabras clave: Condiciones socioeconómicas; Nivel socioeconómico; Situación socioeconómica; Interculturalidad; Pobreza (Fuente: DeCS BIREME).

\section{Socioeconomic and sociodemographic characteristics of the family members of patients hospitalized in the Instituto Nacional de Salud del Niño San Borja, 2018}

\section{ABSTRACT}

Objective: To describe the family and social characteristics of patients hospitalized in the Instituto Nacional de Salud del Niño San Borja from April to December 2018.

Materials and methods: A descriptive, observational and analytical research. Probability sampling was used in a study population of 4,383 patients. The sample consisted of 573 patients, with a $96 \%$ confidence level and a $4 \%$ margin of error. A data collection sheet included in the medical record was used as instrument. The data was processed using the IBM SPSS Statistics statistical software.

1 Universidad Nacional Mayor de San Marcos, Escuela Académica Profesional de Trabajo Social. Lima, Perú.

2 Instituto Nacional de Salud del Niño San Borja. Lima, Perú.

a Licenciada en Trabajo Social, Magister en Política Social con mención en Proyectos Sociales.

b Licenciada en Trabajo Social.

*Autor corresponsal. 
Results: Forty-seven point one two percent (47.12 \%) of the patients came from Lima Metropolitan Area, $2.97 \%$ from the Constitutional Province of Callao, and 49.91 \% from inland, including Lima Province. Seventy-three point six five percent $(73.65 \%)$ of the patients lived in poverty, $24.26 \%$ lived in extreme poverty, and only $2.09 \%$ were not considered poor. Forty point four nine percent $(40.49 \%)$ of the patients lived in unsuitable housing conditions. Most study subjects were holders of the Comprehensive Health Insurance (SIS) (94.24\%). Regarding the mothers' and fathers' educational level, $1.92 \%$ and $0.52 \%$ received no education, and $7.68 \%$ and $4.19 \%$ did not finish primary school, respectively. Forty-three point one one percent $(43.11 \%)$ of the mothers and $39.3 \%$ of the fathers finished the regular basic education (EBR). Nine point seven seven percent $(9.77 \%)$ of the fathers and only $6.63 \%$ of the mothers had higher technical education.

Conclusions: The study enabled us to take a close look to the patients' socioeconomic conditions, which definitely affect their health. It showed that most patients live in poverty and extreme poverty. A significant percentage of the patients come from the different departments of Peru. This situation reflects the centralism prevailing in the specialized pediatric centers and demonstrates the importance of the intercultural approach in health. The type of insurance is related to the patients' economic status. It was confirmed that most of them hold the SIS and live in poverty or extreme poverty. The patients' low economic status is related to dwellings which do not comply with minimum housing standards. This fact is a risk factor for patients with chronic diseases or who got a transplant (bone marrow and solid organs). Finally, fathers evidenced a higher education level than mothers, which could suggest a gender gap.

Keywords: Social class; Social class; Social class; Cultural competency; Poverty (Source: MeSH NLM).

\section{INTRODUCCIÓN}

El Instituto Nacional de Salud del Niño San Borja (INSNSB) es un centro especializado de alta complejidad que atiende a niños, niñas y adolescentes en todo el país en el tratamiento de enfermedades quirúrgicas complejas, trasplantes de órganos, tejidos y células, de acuerdo a ocho ejes: Cirugía Neonatal Compleja, Cardiología y Cirugía Cardiovascular, Neurocirugía, Atención Integral al Paciente Quemado, Trasplante de Médula Ósea, Pediatría y Especialidades Pediátricas, Especialidades Quirúrgicas y Trasplante de Órganos Sólidos.

Como instituto especializado de referencia nacional, la población atendida procede de contextos socioeconómicos y familiares distintos que influyen en su salud. Estos pacientes pediátricos cuentan con el acompañamiento permanente de un familiar o tutor durantela hospitalización. El acompañamiento familiar ha sido materia de estudio en diversas investigaciones, las cuales han evidenciado su importancia en el proceso de atención y recuperación del paciente ${ }^{(1-3)}$.

Otro aspecto que se investiga es la condición socioeconómica de la familia del paciente y su influencia en la condición de salud, como el estudio de Rodríguez ${ }^{(4)}$, que muestra la influencia negativa de la precariedad económica sobre las condiciones de la recuperación de la salud.

Como se pude colegir, considerar los factores socioeconómicos en relación con la salud es tener una mirada holística del proceso salud-enfermedad, que trasciende a lo meramente biológico y toma en cuenta aspectos psíquicos y sociales. Esta perspectiva concuerda con la concepción de salud de la Organización Mundial de la Salud (OMS) ${ }^{(5)}$ que, en su carta fundacional (abril de 1946), define que la salud es el estado de completo bienestar físico, mental, social, y no solo la ausencia de enfermedades o invalideces. Dicha definición se relaciona con el constructo "determinantes de la salud", que toma en cuenta a factores como el ingreso y posición social, las redes de apoyo social, la educación, el empleo y las condiciones de trabajo, los entornos físicos seguros y limpios, las características biológicas y la constitución genética, el desarrollo del niño y los servicios de salud ${ }^{(6)}$.

Considerando las definiciones precedentes, podemos afirmar que las características socioeconómicas de la familia influyen en el desarrollo integral del niño y adolescente; así mismo, la situación de salud de un niño o adolescente tiene impacto en la vida de todos los miembros de la familia. Por ello, es de vital importancia contar con información sobre las características socioeconómicas de los pacientes y sus familias, para coadyuvar en la generación o fortalecimiento de estrategias, políticas, programas y/o lineamientos de atención a los pacientes y sus familias. Al respecto, Kisnerman (7) destaca que es importante obtener los datos que, desde el punto de vista sanitario, se consideran prioritarios para elaborar planes de salud; es decir, que los resultados de las investigaciones son insumos para la planificación de acciones transformadoras.

La mirada holística de la salud lleva a la aplicación de políticas sanitarias de atención centrada en las personas. Nos referimos a la política de atención humanizada, que en los últimos años ha sido impulsada por el Ministerio de Salud del Perú y es un lineamiento de atención al paciente pediátrico en el Instituto Nacional de Salud del Niño San Borja. Oliveros-Donohue ${ }^{(8)}$ resalta que la humanización de los espacios sanitarios es parte del concepto más amplio de humanización en el ámbito de la salud, ya que hace partícipe a los niños hospitalizados en la percepción de un 
Características socioeconómicas y sociodemográficas de las

familias de pacientes hospitalizados en el Instituto Nacional de

Salud San Borja, 2018

ambiente cálido que lo aproxima al recuerdo de su hogar. Así mismo, se privilegia el acercamiento del binomio madre-niño y a todos los involucrados en la asistencia sanitaria.

La percepción del paciente pediátrico sobre un ambiente cálido es de suma importancia en su proceso de atención en salud, como se ha demostrado en estudios internacionales, como el de Herrera-Silva (9), "evitar el maltrato infantil hospitalario" debe ser una prioridad en las políticas de salud. Como vimos en las líneas precedentes, este ambiente cálido implica el buen trato, privilegiar el binomio madre-niño, el respeto a las diferencias culturales y generar estrategias para que el paciente experimente situaciones que le recuerden a su hogar.

\section{MATERIALES Y MÉTODOS}

\section{Población y diseño de estudio}

El diseño de la investigación fue observacional, descriptivo, transversal, retrospectivo. La población de estudio fueron todos los pacientes hospitalizados en los diferentes ejes del Instituto Nacional de Salud del Niño San Borja, desde abril a diciembre del año 2018. La selección de la muestra fue probabilística, el criterio de inclusión para la selección de los pacientes fue contar con la ficha socioeconómica completa. El universo fue de 4383 pacientes y la muestra de 573 , con un nivel de confianza de $96 \%$ y un margen de error de $4,00 \%$.

\section{Variables y mediciones}

El instrumento de recolección de datos fue una sección de la ficha socioeconómica aplicada por los profesionales de Trabajo Social y archivada en la historia clínica de cada paciente. Las variables que contiene dicha ficha social son las siguientes: características del paciente, características del padre, madre $\mathrm{y} / \mathrm{o}$ apoderado, nivel educativo del padre, madre y/o apoderado, tipo de familia de procedencia del paciente, característica de la vivienda del paciente, categoría ocupacional del padre, madre y/o apoderado, condición económica familiar.

\section{Análisis estadístico}

Los datos fueron procesados en el software estadístico IBM SPSS Statistics versión 14, 0. Con ello se diseñaron tablas para la interpretación estadística de los datos, como el análisis de frecuencia, medidas de tendencia central (media, mediana y moda) y de dispersión (desviación estándar y varianza).

\section{Consideraciones éticas}

El estudio respeta los principios de la bioética de autonomía, beneficencia, justicia y no maleficencia. Los datos forman parte de la ficha consignada en la historia clínica. La información recabada fue almacenada con absoluta y total confidencialidad, y fue utilizada solo para fines de investigación, con total anonimato.

\section{RESULTADOS}

\section{Características demográficas de los pacientes}

Del total de pacientes sujetos de la investigación, el $54,97 \%$ fueron hombres y el $45,03 \%$, mujeres. Por ser una institución pediátrica, las edades fluctúan desde recién nacidos (RN), hasta los 17 años, 29 días de edad. Para la investigación fueron agrupados de la siguiente manera: $3,14 \%$, neonatos ( $0-27$ días); $35,43 \%$, lactantes (28 días a 01 año 11 meses); 21,29\%, preescolares (2 años a 5 años 11 meses); 25,31\%, "escolares" (6 a 11 años 11 meses); 14,66 \%, adolescentes (12 años a 17 años 11 meses), y solo se reportó una paciente de 18 años, como caso excepcional, que representa el $0,17 \%$.

Según el lugar de procedencia, el 47,12 \% son de Lima Metropolitana, el 2,97 \% proviene de la Provincia Constitucional del Callao y el $49,91 \%$ son pacientes procedentes del interior del país, incluyendo Lima Provincia.

\section{Condición económica de la familia del paciente}

Uno de los objetivos de la investigación fue conocer la condición económica familiar de los pacientes hospitalizados. Para ello, se tomó la definición de pobreza del Instituto Nacional de Estadística e Informática (INEI) (10), según el cual la pobreza es una condición en la que una o más personas tienen un nivel de bienestar inferior al mínimo aceptado socialmente.

Para medir la pobreza existen diferentes métodos; en esta investigación se utilizó el de Necesidades Básicas Insatisfechas (NBI), que considera un conjunto de indicadores relacionados a las necesidades básicas estructurales (vivienda, educación, salud, infraestructura pública, etc.). Según este método, las personas que tienen por lo menos una NBI son consideradas "pobres", y las que tienen por lo menos dos NBI, "pobres extremos". Los indicadores empleados para medir las NBI son (11)

1. Hogares en viviendas con características físicas inadecuadas: toma en cuenta el material predominante en las paredes y pisos, así como al tipo de vivienda.

2. Hogares en viviendas con hacinamiento

3. Hogares en viviendas sin desagüe de ningún tipo

4. Hogares con niños que no asisten a la escuela

5. Hogares con alta dependencia económica.

Bajo este enfoque de medición de la pobreza, se identificó que el 73,65\% de pacientes tiene condición de pobreza; el 24,26 \% están en condición de pobreza extrema, y solo el $2,09 \%$ son considerados no pobres. (Figura 1 ) 


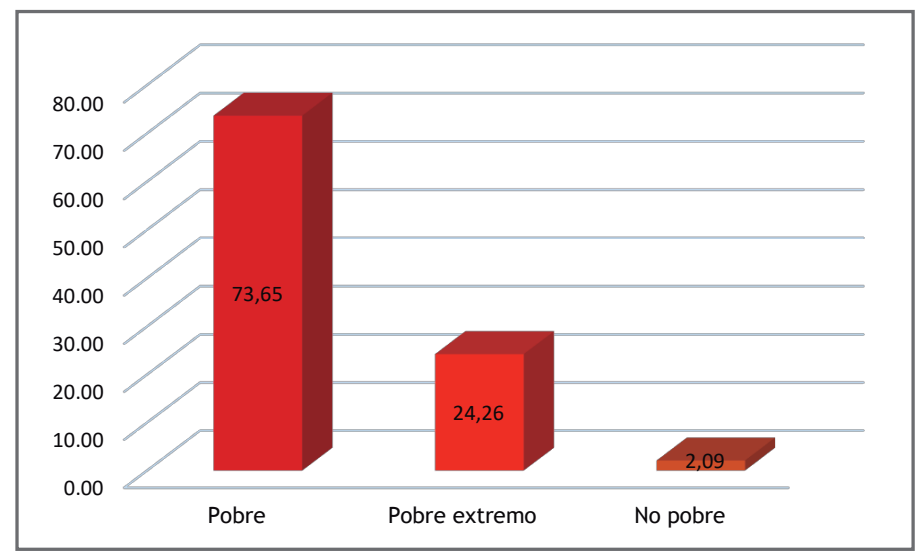

Figura 1. Condición económica

\section{Tipo de seguro del paciente}

El 94,24\% de los pacientes hospitalizados son beneficiarios del Seguro Integral de Salud (SIS). El 3,67 \% cuentan con otros seguros de salud como el Seguro Social (EsSalud), el seguro contra accidentes de tránsito (SOAT) y el seguro de las sanidades (Fondo de la Sanidad de la FAP, de la Marina y del Ejército). El 2,09 \% no cuentan con algún seguro de salud. Con el cruce estadístico de las variables tipo de seguro de paciente y condición económica, se identificó que el tipo de seguro prevalente es el SIS, correspondiente al $69,98 \%$ de las personas en situación de pobreza y al 23,56 \% de pacientes en situación de pobreza extrema.

Tabla 1. Tipo de seguro de paciente*Condición económica

\begin{tabular}{|c|c|c|c|c|c|c|c|c|}
\hline & & \multicolumn{7}{|c|}{$\begin{array}{l}\text { Tabla cruzada Condición económica* Tipo de seguro de paciente } \\
\qquad \text { Tipo de seguro de paciente }\end{array}$} \\
\hline & & & No tiene & SIS & EsSalud & SOAT & Sanidades & Total \\
\hline Condición & Pobre & Recuento & 9 & 401 & 10 & 2 & 0 & 422 \\
\hline \multirow[t]{5}{*}{ económica } & & $\%$ del total & $1,57 \%$ & $69,98 \%$ & $1,75 \%$ & $0,35 \%$ & $0,00 \%$ & $73,65 \%$ \\
\hline & Pobre & Recuento & 2 & 135 & 2 & 0 & 0 & 139 \\
\hline & extremo & $\%$ del total & $0,35 \%$ & $23,56 \%$ & $0,35 \%$ & $0,00 \%$ & $0,00 \%$ & $24,26 \%$ \\
\hline & No pobre & Recuento & 1 & 4 & 5 & 0 & 2 & 12 \\
\hline & & $\%$ del total & $0,17 \%$ & $0,70 \%$ & $0,87 \%$ & $0,00 \%$ & $0,35 \%$ & $2,09 \%$ \\
\hline \multirow[t]{2}{*}{ Total } & & Recuento & 12 & 540 & 17 & 2 & 2 & 573 \\
\hline & & $\%$ del total & $2,09 \%$ & $94,24 \%$ & $2,97 \%$ & $0,35 \%$ & $0,35 \%$ & 100,00 \\
\hline
\end{tabular}

\section{CARACTERÍSTICAS DE LOS PROGENITORES DEL PACIENTE}

\section{Rangos de edad de la madre y el padre}

Las madres menores de edad (12 a 17 años) representan un porcentaje mínimo de 2,09\%; el rango de edad entre 18 a 29 años equivale al $36,47 \%$; las madres de 30 a 44 años representan el 51,83 \%; entre los 45 a 64 años constituyen el $8,03 \%$, y finalmente, las de 65 a más años son el $0,17 \%$. Cabe señalar que el $1,40 \%$ de madres no brindaron esta información.

Por su parte, los padres menores de edad, también, representan un porcentaje minoritario $(1,05 \%)$; entre los 18 a 29 años representan el 24,61\%; los de 30 a 44 años son el 47,82\%; el grupo entre 45 a 64 años constituye el $16,93 \%$, y por último, los padres de 65 a más años representan el 0,52 \%. Cabe señalar que el 9,08\% de padres no dieron este dato.

Al comparar ambas variables, se encontró que en el grupo etario de 30-44 años, el porcentaje de mujeres es mayor $(51,83 \%)$ en comparación a los hombres $(47,82 \%)$. Mientras que, en el grupo etario de 12 a 17 años, el 2,09\% corresponde a mujeres, y solo el 1,05\%, a varones (Figura 2). 
Características socioeconómicas y sociodemográficas de las

familias de pacientes hospitalizados en el Instituto Nacional de

Salud San Borja, 2018

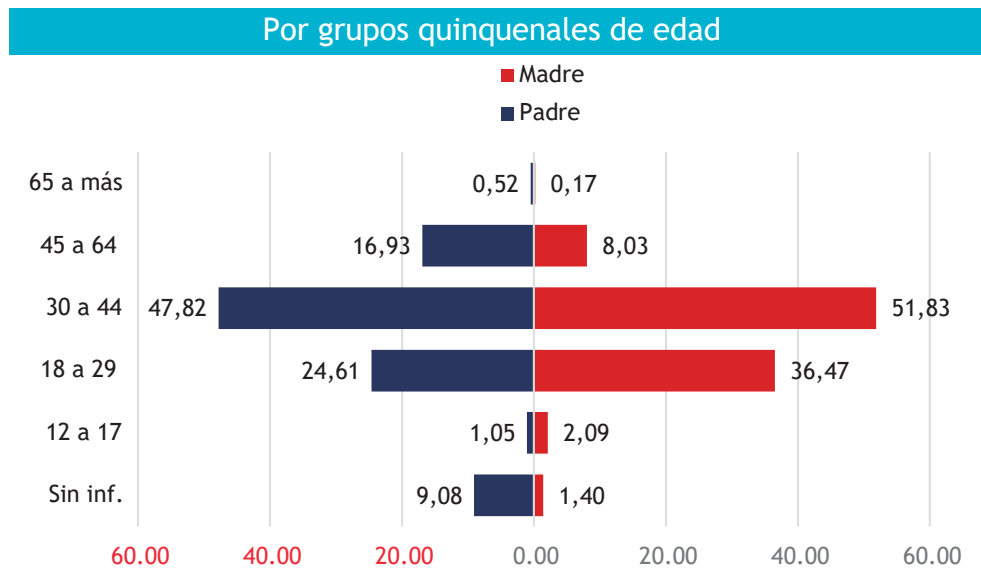

Figura 2. Edad de padres y madres de pacientes

\section{Grado de instrucción de los padres del paciente}

Para analizar el grado de instrucción de las madres de familia, se las agrupó según el nivel educativo: las progenitoras con "nivel bajo" de instrucción representan el 21,47 \% (sin instrucción y estudios de nivel primaria), y llama la atención que el 1,92 \% de madres no tiene instrucción, es decir, nunca asistió a la escuela, por lo cual son iletradas. Otro grupo está constituido por las madres con "nivel medio" de instrucción, que representa el 60,21 \% (secundaria incompleta y completa). Por último, las progenitoras con "alto nivel" de instrucción representan el 16,41\% (nivel superior técnico o universitario completo o incompleto). Cabe señalar que el 1,92\% de las madres no brindaron esta información.

La instrucción de los padres de familia también fue analizada según niveles: los padres con bajo nivel de instrucción representan el 14, $66 \%$, dentro de los cuales el porcentaje de padres sin instrucción es minoritario $(0,52 \%)$. Los padres de familia con nivel medio de instrucción constituyen el 54,63\%. Finalmente, el 19,37\% tiene estudios superiores técnicos o universitario completo o incompleto.

Al comparar ambos resultados, se observó que el $43,11 \%$ de mujeres tienen estudios de secundaria completa, a diferencia de los hombres, de quienes solo 39,27 \% terminaron la secundaria completa. El 9,77 \% del grupo de varones tienen superior técnica y el 6,63\% de mujeres cursaron superior técnica. El 1,92\% de mujeres no tienen grado de instrucción a comparación del grupo de varones que representa el 0,52 \% (Figura 3).

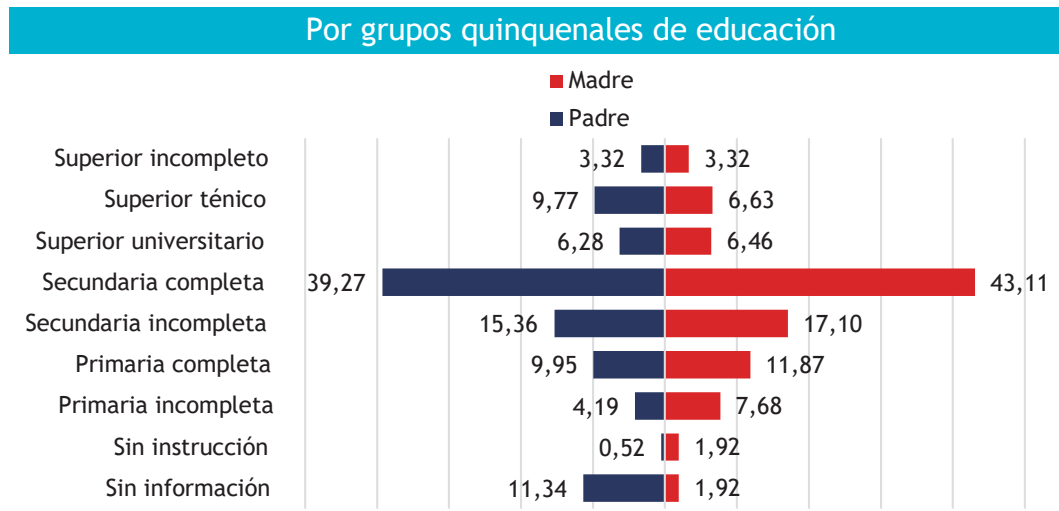

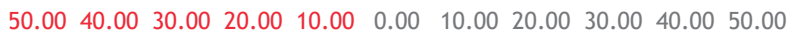

Figura 3. Grado de instrucción de padres y madres 


\section{Ocupación del jefe de hogar}

Se identificó que el $57,59 \%$ de jefes de hogar de los pacientes hospitalizados son obreros; el 16,75\%, campesinos, y el $3,49 \%$, trabajadoras del hogar.

El 57,59\% de los jefes del hogar se encuentran en situación de pobreza, mientras que el 16,75\% de campesinos viven en situación de pobreza y pobreza extrema, lo cual se relaciona con reportes nacionales que señalan que en las zonas rurales se concentra la mayor cantidad de personas en condición de pobreza. Por otro lado, los resultados revelan que los técnicos $(5,41 \%)$ y profesionales $(1,40 \%)$ se encuentran en condición de pobreza, lo cual refleja que contar con un trabajo no implica superación de esta (Tabla 2).

Tabla 2. Tabla cruzada Tipo de ocupación del jefe de hogar *Condición económica

\begin{tabular}{|c|c|c|c|c|c|c|}
\hline & & & \multicolumn{4}{|c|}{ Condición económica } \\
\hline & & & Pobre & Pobre extremo & No pobre & Total \\
\hline Tipo de & Sin información & Recuento & 1 & 1 & 0 & 2 \\
\hline ocupación del & & $\%$ del total & $0,17 \%$ & $0,17 \%$ & $0,00 \%$ & $0,35 \%$ \\
\hline \multirow[t]{14}{*}{ jefe de hogar } & Profesional & Recuento & 5 & 0 & 3 & 8 \\
\hline & & $\%$ del total & $0,87 \%$ & $0,00 \%$ & $0,52 \%$ & $1,40 \%$ \\
\hline & Técnico profesional & Recuento & 22 & 5 & 4 & 31 \\
\hline & & $\%$ del total & $3,84 \%$ & $0,87 \%$ & $0,70 \%$ & $5,41 \%$ \\
\hline & Comerciante & Recuento & 72 & 12 & 2 & 86 \\
\hline & & $\%$ del total & $12,57 \%$ & $2,09 \%$ & $0,35 \%$ & $15,01 \%$ \\
\hline & Trabajador del hogar & Recuento & 5 & 15 & 0 & 20 \\
\hline & & $\%$ del total & $0,87 \%$ & $2,62 \%$ & $0,00 \%$ & $3,49 \%$ \\
\hline & Campesino & Recuento & 41 & 55 & 0 & 96 \\
\hline & & $\%$ del total & $7,16 \%$ & $9,60 \%$ & $0,00 \%$ & $16,75 \%$ \\
\hline & Obrero & Recuento & 276 & 51 & 3 & 330 \\
\hline & & $\%$ del total & $48,17 \%$ & $8,90 \%$ & $0,52 \%$ & $57,59 \%$ \\
\hline & Total & Recuento & 422 & 139 & 12 & 573 \\
\hline & & $\%$ del total & $73,65 \%$ & $24,26 \%$ & $2,09 \%$ & $100,00 \%$ \\
\hline
\end{tabular}

\section{CARACTERÍSTICAS SOCIOECONÓMICAS DE LA FAMILIA}

\section{Tipo de familia}

El 66,14\% de los pacientes provienen de familias nucleares (padres e hijos); y el 15,53\%, de familias monoparentales (separación de los padres, abandono, divorcio, etc.). El $10,82 \%$ de los pacientes provienen de familias reconstituidas que están integradas por una pareja en la que uno o ambos miembros han tenido otras parejas y han disuelto el vínculo marital. En la nueva familia es frecuente que los hijos sean de diferentes padres o madres. El 6,46\% son familias extensas (pareja con o sin hijos y otros miembros como sus parientes consanguíneos ascendentes, descendientes, recogen varias generaciones). El 1,05\% son familias ampliadas (miembros no consanguíneos o convivientes afines, tales como vecinos, colegas, paisanos, etc.).

\section{Características de la vivienda}

Más de la mitad de los pacientes $(55,50 \%)$ habita en viviendas con paredes construidas con material adecuado. Otro porcentaje importante $(45,50 \%)$ vive en casas de paredes hechas con material inadecuado, es decir, adobe/tapia, quincha/piedra en barro, madera. Respecto al material de construcción del techo, se observa que el 57,94\% habitan en casas construidas con techo de material inadecuado. Respecto al piso de la vivienda, el 59,51 \% de los casos, las casas tienen pisos construidos de manera adecuada; y el $40,49 \%$ ocupa viviendas con pisos de material inadecuado, lo cual también es un indicador de medición de la pobreza.

\section{Tenencia y tipo de vivienda}

Respecto a la tenencia de la vivienda, la mayoría de los pacientes $(38,22 \%)$ habita una vivienda que no es de propiedad de sus progenitores, a lo cual denominamos "alojados". Es decir, la propiedad es de algún familiar que los acoge en su vivienda, ya sea que les proporciona un espacio común o un ambiente de uso exclusivo para la familia. El 35,43\% de pacientes habitan viviendas de propiedad de sus progenitores. Según el tipo de vivienda 
que habitan, el $70,33 \%$ de pacientes ocupan viviendas de tipo unifamiliar, y el $29,67 \%$, viviendas multifamiliares.

\section{Acceso a servicios de agua y desagüe}

El 75,22 \% de los pacientes tiene acceso a la red pública de desagüe y el $23,21 \%$ que no utiliza este servicio emplea medios alternos de alcantarillado, tales como letrina o pozo séptico. A pesar de ser minoritario (1,57\%), es importante resaltar que un grupo de pacientes no utiliza la red de alcantarillado, lo cual se relaciona con los pacientes en extrema pobreza. La mayoría de los pacientes $(83,94 \%)$ acceden al servicio de agua potable por red pública. El $8,55 \%$ de pacientes consume agua no potable, y recolecta el agua directamente de fuentes naturales como el río o el subsuelo. El 7,51 \% de pacientes sí accede a agua potable, pero no de red pública, sino que se abastece de un camión cisterna o de alguna vivienda aledaña (Tabla 3).

Tabla 3. Tabla cruzada Acceso a servicio de agua*Acceso a servicio de desagüe

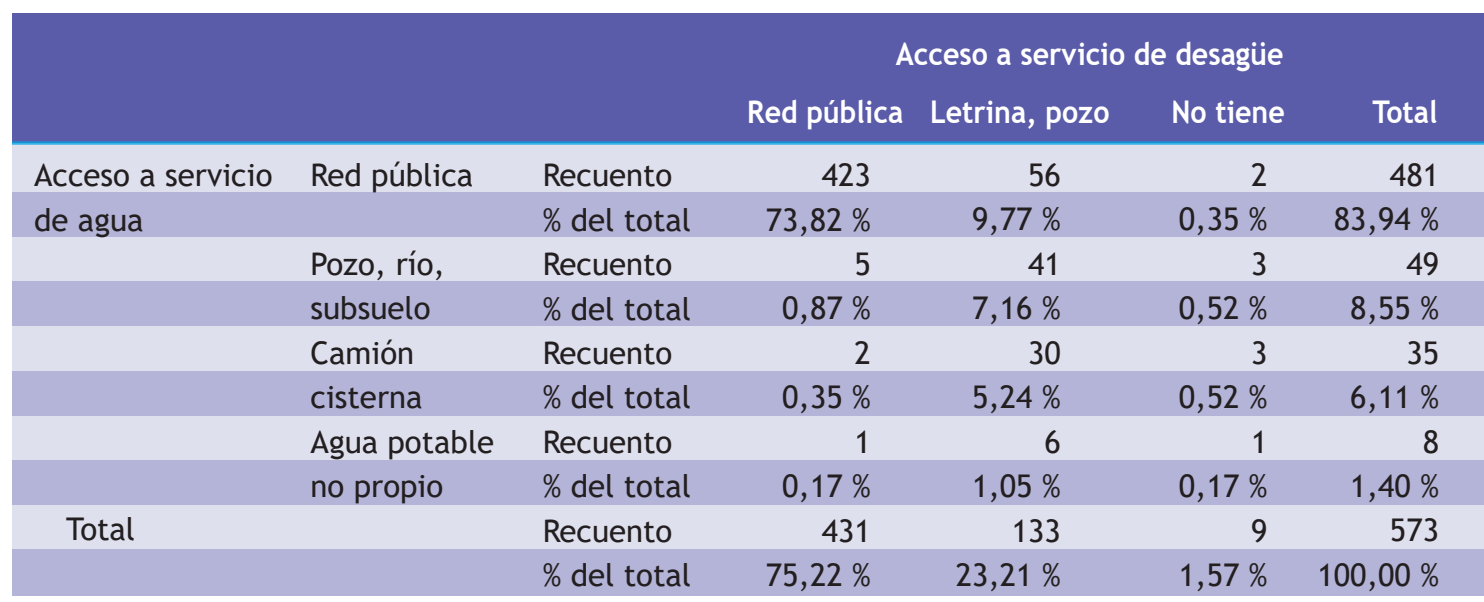

\section{Acceso a servicios de alumbrado}

El 94,42\% de los pacientes utiliza energía eléctrica en sus hogares; de ellos, el 5,41\% declara que no es el titular de la luz eléctrica que consume. El 5,59\% de pacientes utiliza energía proveniente de otras fuentes como combustible $(0,87 \%)$, vela $(2,97 \%)$, linterna $(1,05 \%)$ y panel solar $(0,70 \%)$. Se puede inferir que el acceso a servicio eléctrico es una necesidad satisfecha para la mayoría, pero también se observa indicadores de pobreza extrema en zonas rurales.

\section{DISCUSIÓN}

\section{Sobre la condición socioeconómica del paciente}

El estudio evidencia que la mayoría de los pacientes atendidos en el Instituto Nacional De Salud del Niño San Borja viven en situación de pobreza $(73,65 \%$ en pobreza y $24,26 \%$ en pobreza extrema). Estos resultados pueden motivar nuevas investigaciones en la institución que correlacionen el estado de salud con la condición económica, como el estudio de Vera-Romero ${ }^{(12)}$, cuya conclusión es que existe evidencia de la relación entre el nivel socioeconómico y el estado de salud.

Bajo esta misma línea, destaca el estudio del Instituto Nacional de Salud que encuentra que el nivel socioeconómico del hogar es una variable estadísticamente significativa para explicar el nivel de desnutrición existente y los episodios de enfermedad, ya que las familias más pobres son las que muestran una persistencia mayor de desnutrición o enfermedad ${ }^{(13)}$.

Los resultados del estudio sobre la condición socioeconómica del paciente pueden coadyuvar a la generación $\mathrm{y} / \mathrm{o}$ fortalecimiento de estrategias, políticas y programas institucionales de atención social e interdisciplinaria a los pacientes y sus familias. Así mismo, permite valorar la importancia del trabajo interdisciplinario, donde la intervención del profesional en Trabajo Social toma relevancia en el ámbito social con las familias y aporta al equipo multidisciplinario en la investigación social de variables socioeconómicas y culturales ${ }^{(7)}$.

\section{Sobre el lugar de procedencia y condición económica de los pacientes}

Según Alcalde-Rabanal ${ }^{(14)}$, alrededor de $90 \%$ de hospitales e institutos de salud del MINSA en todo el país se ubican en zonas urbanas, esto implica que población de jurisdicciones rurales en condición de pobreza y pobreza extrema debe trasladarse hacia la capital para conseguir la atención especializada, lo que genera mayores gastos para la precaria economía familiar. Esta situación refleja el centralismo nacional en torno a la ciudad de Lima, donde se encuentran los institutos pediátricos especializados. 
El 47,12 \% de los pacientes vienen de Lima Metropolitana; el 2,97\%, de Callao; y el 49,91\%, de las provincias (incluyendo Lima Provincia). Las estadísticas muestran que todos los pacientes que vienen de provincia se encuentran en condición de pobreza y pobreza extrema, lo cual implica que la lejanía a su lugar de origen ocasiona dificultades para solventar los gastos adicionales a la atención médica, como el transporte y, en general, los costos de estadía en la capital.

Esta situación ha generado que el Instituto Nacional de Salud del Niño San Borja aplique programas de subvención en alimentación y alojamiento para familiares de un paciente que viene de provincia. Para el alojamiento, se cuenta con un albergue institucional para madres de los niños hospitalizados en las Unidades de Cuidados Intensivos ${ }^{(15)}$.

En el caso de la alimentación, todos los acompañantes de los pacientes hospitalizados procedentes de provincia son beneficiados con desayuno, almuerzo y cena. Esto responde a la "Política de Humanización en Salud", con lo cual se pretende brindar mejores condiciones para que las familias afronten el proceso de atención médica especializada.

Por otro lado, es importante considerar las características culturales de los pacientes procedentes de zonas rurales, tales como el lenguaje, costumbres, alimentación, etc. Según los documentos normativos que sustentan la Política Nacional para la Transversalización del Enfoque Intercultural en el Sector Salud, la interculturalidad es entendida como la habilidad para reconocer, armonizar y negociar las múltiples diferencias que existen al interior de cada sociedad. El objetivo de esta política es garantizar el ejercicio de los derechos de la población culturalmente diversa del país, sobre todo de los pueblos indígenas y la población afroperuana, promoviendo un Estado que reconoce la diversidad cultural innata de nuestra sociedad, opera con pertinencia cultural y contribuye así a la inclusión social, la integración nacional y la eliminación de la discriminación (16-18).

Concordante con esta Política de Transversalización de la Interculturalidad, en el Instituto Nacional de Salud del Niño San Borja se han implementado iniciativas de innovación desde un enfoque de interculturalidad en salud, como es el caso de los programas "Hablamos el mismo idioma", desarrollado por el área de Trabajo Social, y "Comida como en casa", del área de Nutrición. Ambos han sido reconocidos como buenas prácticas por la asociación Ciudadanos al Día (19-20).

\section{Sobre el seguro de salud}

El tipo de seguro de los pacientes hospitalizados se relaciona con su condición económica, y se corrobora que la mayoría de los pacientes beneficiarios del Seguro Integral de Salud (SIS) tienen condición de pobreza y pobreza extrema. Cabe señalar que, a pesar de que el SIS cubre los costos de la atención, hay gastos adicionales como el traslado y estadía que deben ser asumidos por la familia, lo cual genera dificultades para su precaria condición económica.

El estudio evidenció un porcentaje mínimo de personas beneficiarias del SIS categorizadas como "no pobres". Esta situación podría explicarse como "filtración" en el Sistema de Focalización de Hogares (SISFOH), es decir, el acceso de personas no pobres o no pobres extremos a programas sociales focalizadas. Los errores en la focalización han sido abordados en sendos estudios, como el de Olivares ${ }^{(21)}$, quien describe elevadas cifras de filtración y subcobertura en la identificación de beneficiarios de programas sociales. En la misma línea se encuentra la investigación de Simonetti ${ }^{(22)}$, sobre la focalización para la afiliación al Seguro Integral de Salud. A pesar de los errores minoritarios en la focalización, se puede concluir que el Seguro Integral de Salud (SIS) es el principal soporte económico durante la atención médica de los pacientes en condición es de pobreza y pobreza extrema.

Respecto a los pacientes beneficiarios del Seguro Social EsSalud, se evidencia que la mayoría son de condición económica "no pobres"; sin embargo, existe un mínimo porcentaje categorizado como "pobres". Esta situación se puede explicar porque el salario mínimo vital no asegura que una familia con alto grado de dependencia pueda cubrir todas las necesidades básicas (hogares con más de tres personas por cada persona ocupada o perceptora).

\section{Sobre el nivel educativo de los progenitores de los pacientes}

La estadística muestra que la mayoría de madres de familia $(43,11 \%)$ ha concluido los estudios de educación básica regular. Un porcentaje de progenitoras que tienen bajo nivel de instrucción $(21,47 \%)$, que incluye a las que solo tiene estudios de primaria y a las que son iletradas $(1,92 \%)$. Aunque es un porcentaje bajo, debe considerarse para orientar los lineamientos específicos dirigidos a este grupo, por ejemplo, cuando se les brinda informes médicos o cuando firman los consentimientos informados, lo cual requiere una atención diferenciada por su condición de personas iletradas.

El $14,66 \%$ de padres de familia tiene bajo nivel de instrucción, este porcentaje es menor que el de las madres de familia con bajo nivel de instrucción, lo que podría sugerir una brecha de género en el aspecto educativo entre padres y madres.

El $39,00 \%$ de los padres de familia han concluido los estudios de secundaria y el $16,00 \%$ tiene estudios superiores, entre universitarios y técnicos. Esto podría ser considerado una fortaleza si se cuenta con la presencia y participación del padre. También se identificó que la mayoría de los 
Características socioeconómicas y sociodemográficas de las

familias de pacientes hospitalizados en el Instituto Nacional de

Salud San Borja, 2018

progenitores ha concluido la educación básica regular; sin embargo, existe un porcentaje minoritario de progenitores iletrados y con primaria incompleta, lo cual debe tomarse en cuenta para la adecuación de contenidos y estrategias educativas.

Este resultado se condice con otras investigaciones que resaltan la importancia del nivel educativo de los padres en la preservación de la salud del menor, tanto en el aspecto preventivo como en la atención recuperativa. El estudio de Loyola ${ }^{(23)}$ concluye que la madre es la principal proveedora de información para el niño, por lo cual es importante conocer las características sociodemográficas y conductuales de ella para el diseño de programas preventivos.

Conocer el nivel educativo de madres y padres permite orientar acciones socioeducativas considerando su nivel educativo, promoviendo el uso de estrategias de comunicación y técnicas de educación social adecuadas.

\section{Sobre la condición laboral de los progenitores del paciente}

El 57,59 \% de jefes de hogar son "obreros", esta categoría ocupacional es definida por el Ministerio de Trabajo como la persona que desempeña actividades de carácter manual, que trabaja para un empleador público o privado, y que percibe, por lo general, una remuneración semanal.

El 16,75\% de jefes del hogar son campesinos, esta condición laboral guarda relación con la cantidad de pacientes procedentes de las diferentes regiones del país, desde zonas rurales donde una de las principales actividades económicas es la agricultura.

El 3,49\% de jefes del hogar manifiesta que tiene un trabajo familiar no remunerado; se trata de madres de familia que constituyen familias monoparentales, donde ellas son el soporte del hogar, pero no realizan labor remunerada y se dedican a labores domésticas al servicio de otros familiares que, a cambio, les brindan alimentos y materiales para la manutención de sus hijos. Es importante considerar esta situación para visibilizar el trabajo doméstico intrafamiliar como una forma de generar ingresos de manera indirecta, a pesar de que las personas entrevistadas que perciben que ellas realizan las labores domésticas a cambio de la "ayuda de sus familiares", este tipo de actividad no es considerada como un "trabajo", sino como parte de las relaciones familiares de "ayuda mutua". En la práctica, sin embargo, se genera un valor monetario no visibilizado.

\section{Sobre el tipo de familia}

Los datos muestran que más de la mitad de los pacientes proviene de familias nucleares $(66,14 \%)$. Esta condición brinda mejores posibilidades de involucramiento consensuado de los progenitores en el tratamiento del paciente, aunque no es una determinante. Las familias extensas y ampliadas alcanzan un menor porcentaje $(7,51 \%)$.

El 10,82\% de pacientes proviene de familias reconstituidas, es decir, formadas por parejas que proceden de una relación anterior disuelta. En este tipo de familias es importante promover y/o fortalecer el consenso de responsabilidades entre los progenitores biológicos para afrontar el proceso de enfermedad del paciente.

\section{Sobre las características de la vivienda}

El acceso a la vivienda es una necesidad básica que debe tener condiciones aceptables, en cuanto a la habitabilidad, la privacidad y el confort mínimo de los miembros del hogar. Las condiciones de vivienda son consideradas como determinantes de la salud. Para el Ministerio de Salud, "las condiciones de la vivienda han sido reconocidas como una de las principales determinantes sociales de la salud humana. Las condiciones de la vivienda pueden promover o limitar la salud física, mental y social de sus residentes" (24) y, de acuerdo con las Necesidades Básicas Insatisfechas, las características físicas inadecuadas (material predominante en las paredes y pisos, así como el tipo de vivienda) son indicadores para medir la pobreza.

El Ministerio de Vivienda clasifica la infraestructura de una casa según el material de construcción predominante: material de primera (ladrillo o bloques de cemento) que, para efectos de la investigación, se ha catalogado como "adecuada"; material de segunda (adobe) y material de tercera u otro material (madera, paja, caña), los cuales son catalogados como "inadecuados". Según los datos analizados, casi la mitad de las viviendas son calificadas como inadecuadas, es decir, no cumplen con los estándares establecidos por el Ministerio de Vivienda. Esto puede influir en la recuperación poshospitalaria, ya que representa un factor de riesgo para pacientes con enfermedades crónicas o postrasplantados (médula ósea y órganos sólidos). Sumado a esto, las condiciones de salubridad en la vivienda son importantes y, en tal sentido, la labor de educación social toma relevancia.

Agradecimientos: A la Dra. María Cristina Medina Pfluker, por su asesoría técnica desde la formulación del proyecto de investigación, hasta la concreción del presente artículo, así como su motivación constante. A Jackelin Miranda Rivera Hurtado y a Vanessa Isabel Torres Damian, por su apoyo en la codificación de los datos en el programa estadístico.

Contribución de las autoras: IAGP: Concepción y diseño del trabajo, recolección de datos, análisis e interpretación de resultados y redacción del manuscrito. GCH: Recolección de datos, análisis e interpretación de resultados y redacción del manuscrito. APS: Análisis e interpretación de resultados y redacción del manuscrito. 
Fuente de financiamiento: Este artículo ha sido financiado por las autoras, con el apoyo logístico del Instituto Nacional de Salud del Niño San Borja, así como la dotación de horas de investigación durante la jornada laboral.

\section{Conflicto de interés: Las autoras declaran no tener ningún conflicto de interés.}

\section{REFERENCIAS BIBLIOGRÁFICAS}

1. Astudillo AA, Martínez AM, Badillo CM, Lema MP, Garay AS. Acompañamiento familiar en la hospitalización del usuario pediátrico de 6 a 12 años. Cienc Enferm. 2012; 18(1): 67-75.

2. Lagos YLA, Rozo CYS, Albarracín DGE. Vivencias de los padres de niños hospitalizados en la unidad de recién nacidos de dos instituciones de salud de la ciudad de Bogotá. Actual Enferm. 2005; 8(2): 8-13.

3. De Prado EMF. Situación socioeconómica del paciente con insuficiencia renal crónica del Instituto Guatemalteco de Seguridad Social [Tesis]. Guatemala: Universidad de San Carlos de Guatemala. Escuela de Trabajo Social; 2003.

4. Rodríguez GJ, Córdoba-Doña JA, Escolar-Pujolar A, Aguilar-Diosdado M, Goicolea I. Familia, economía y servicios sanitarios: claves de los cuidados en pacientes con diabetes y amputación de miembros inferiores. Estudio cualitativo en Andalucía. Aten Primaria. 2018; 50(10): 611-20.

5. Moreno GA. La definición de salud de la Organización Mundial de la Salud y la interdisciplinariedad. Sapiens. 2008; 9(1): 93-107.

6. Lip C, Rocabado F. Determinantes sociales de la salud en Perú [Internet]. Ministerio de Salud: Lima; 2005. p. 84. Disponible en: http://bvs.minsa.gob.pe/local/MINSA/143_detersoc.pdf

7. Kisnerman N. Salud Pública y Trabajo Social. 3ra ed. Argentina: Editorial Humanitas; 1978. p.246.

8. Oliveros-Donohue MA. Humanización de la Pediatría. Acta Méd Peruana. 2015; 32(2): 85-90.

9. Herrera-Silva JC, Treviño-Moore A, Guzmán-Rentería M, AcostaCorona CG. Maltrato hospitalario a escolares y adolescentes con padecimientos crónicos. Bol Med Hosp Infant Mex. 2006; 63(2): 94106.

10. Ministerio de Economía y Finanzas. Métodos para medir la pobreza [Internet]. MEF: Lima; 2009. Disponible en: https://www.mef.gob. pe/es/mapas-de-pobreza/metodos-para-medir-la-pobreza

11. Instituto Nacional de Estadística e Informática. Necesidades básicas insatisfechas de los hogares [Internet]. INEI: Lima; 1993. Disponible en: http://proyectos.inei.gob.pe/web/biblioineipub/bancopub/ Est/Lib0068/n00.htm

12. Vera-Romero OE, Vera-Romero FM. Evaluación del nivel socioeconómico: presentación de una escala adaptada en una población de Lambayeque. Rev Cuerpo Méd HNAAA. 2013; 6(1): 41-5.

13. Serna JP, Cuadros MM, Abanto JS, Aspilcueta HT, Ruiz RS. Efecto del nivel socioeconómico sobre algunos indicadores de salud y nutrición en la niñez, Perú 2003-2004 [Internet]. Instituto Nacional de Salud: Lima; 2007. Disponible en: https://repositorio.ins.gob.pe/handle/ INS/230

14. Alcalde-Rabanal JE, Lazo-González 0 , Nigenda G. Sistema de salud de Perú. Salud Pública Mex. 2011: 53(Suppl. 2): S243-54.

15. Instituto Nacional del Niño San Borja. INSN San Borja destaca albergue para madres de pacientes promoviendo una atención humanizada [Internet]. INSN: Lima; 2021. Disponible en: http://www.insnsb. gob.pe/blog/2018/08/10/insn-san-borja-destaca-albergue-paramadres-de-pacientes-promoviendo-una-atencion-humanizada/

16. Ministerio de Salud. Resolución Ministerial N ${ }^{\circ} 638-2006 /$ MINSA

Norma Técnica de salud para la transversalización de los enfoques de derechos humanos, equidad de género e interculturalidad en salud. Ministerio de Salud: Lima; 2006.

17. Ministerio de Cultura. Decreto Supremo N 003-2015-MC. Decreto Supremo que aprueba la política nacional para la Transversalización del enfoque intercultural. Ministerio de Cultura: Lima; 2015.

18. Presidencia de la República. Decreto Supremo N 016-2016-SA. Política Sectorial de Salud Intercultural. Presidencia de la República: Lima; 2016.

19. Instituto Nacional del Niño San Borja. INSN San Borja es premiado por buenas prácticas interculturales en gestión pública [Internet]. INSN: Lima: 2021. Disponible en: http://www.insnsb.gob.pe/ blog/2017/12/14/insn-san-borja-es-premiado-por-buenaspracticas-interculturales-en-gestion-publica/

20. Instituto Nacional del Niño San Borja. INSN San Borja destaca con buenas prácticas a favor de los pacientes y su entorno en concurso de ciudadanos al día [Internet]. INSN: Lima; 2021. Disponible en: http: / / www.insnsb.gob.pe/blog/2019/10/01/insn-san-borjadestaca-con-buenas-practicas-a-favor-de-los-pacientes-y-suentorno-en-concurso-de-ciudadanos-al-dia/

21. Olivares Ramírez AO. El gasto social del estado: Conceptos y análisis sobre focalización [Tesis]. Callao: Universidad Nacional del Callao. Escuela de Posgrado; 2006.

22. Simonetti Paredes HJ. Análisis de la focalización, afiliación y cobertura del seguro integral de Salud en el distrito de Pampas de hospital, Tumbes 2017 [Tesis]. Piura: Universidad César Vallejo. Escuela de Posgrado; 2019.

23. Loyola MC. Características socioeconómicas y salud bucal de escolares de institucional educativas públicas. Rev Kiru. 2009; 6(2).

24. Ministerio de Salud. Guía de implementación de familias y viviendas saludables [Internet]. Minsa: Lima; 2006. Disponible en: https:// www.gob.pe/institucion/minsa/informes-publicaciones/353434guia-de-implementacion-de-familias-y-viviendas-saludable

\section{Correspondencia:}

Isabel Andrea Gantu Palacios

Dirección: Jr. Guillermo Ramsey 422, Distrito San Martín de

Porres. Lima, Perú.

Teléfono: 949621835

Correo electrónico: igantup@unmsm.edu.pe

Recibido: 28 de septiembre de 2021.

Evaluado: 06 de octubre de 2021

Aprobado: 11 de noviembre de 2021.

(c) La revista. Publicado por Universidad de San Martín de Porres, Perú. (c) Br Licencia de Creative Commons Artículo en acceso abierto bajo términos de Licencia Creative Commons Atribución 4.0 Internacional. (http://creativecommons.org/licenses/by/4.0/)

\section{ORCID iDs}

Isabel Andrea Gantu Palacios Thttps: / / orcid.org/0000-0001-7451-8484 Giovana Genoveva Ccasani Huamán ำ https://orcid.org/0000-0002-9441-8373 Ana Francisca Pérez Solis (1) https: / / orcid.org/0000-0002-4036-6440 